Acta Crystallographica Section E

Structure Reports

Online

ISSN 1600-5368

\section{2-Amino-4,6-dimethylpyridinium benzoate}

\section{Mohd Razip Asaruddin, ${ }^{a}$ Habibah A Wahab, ${ }^{b} \neq$ Nornisah Mohamed, ${ }^{a}$ Mohd Mustaqim Roslic and Hoong-Kun Fun $^{\mathrm{C} * \S}$}

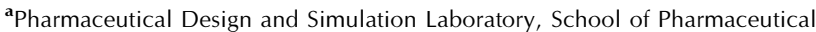
Sciences, Universiti Sains Malaysia, 11800 USM, Penang, Malaysia, $\mathbf{b}$ Institute of Pharmaceutical and Neutraceuticals, Malaysia Ministry of Science and Technology and Innovation, Science Complex, 11900, Malaysia, and ${ }^{\mathbf{C} X-r a y ~ C r y s t a l l o g r a p h y}$ Unit, School of Physics, Universiti Sains Malaysia, 11800 USM, Penang, Malaysia Correspondence e-mail: hkfun@usm.my

Received 17 August 2010; accepted 29 August 2010

Key indicators: single-crystal X-ray study; $T=296 \mathrm{~K}$; mean $\sigma(\mathrm{C}-\mathrm{C})=0.003 \AA$; $R$ factor $=0.044 ; w R$ factor $=0.127 ;$ data-to-parameter ratio $=11.7$.

In the title compound, $\mathrm{C}_{7} \mathrm{H}_{11} \mathrm{~N}_{2}{ }^{+} \cdot \mathrm{C}_{7} \mathrm{H}_{5} \mathrm{O}_{2}{ }^{-}$, the 2-amino-4,6dimethylpyridinium cation and the benzoate anion are linked by two $\mathrm{N}-\mathrm{H}$... O hydrogen bonds, forming an $R_{2}^{2}(8)$ ring motif. The $\mathrm{H}$ atoms in both the methyl groups are rotationally disordered, with fixed site occupancies of 0.50 . In the crystal structure, the molecules are stabilized by intermolecular $\mathrm{N}-$ $\mathrm{H}$.. O hydrogen bonds. A $\pi-\pi$ interaction, with a centroidcentroid distance of 3.661 (2) $\AA$, is also observed.

\section{Related literature}

For the biological activity of Schiff bases with azomethine linkages, see Dhar \& Taploo (1982). For hydrogen bonding, see: Jeffrey (1997); Jeffrey \& Saenger (1991). For graph-set descriptions of hydrogen-bond ring motifs, see: Bernstein et al. (1995).

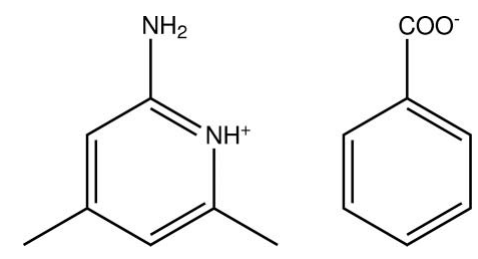

\section{Experimental}

Crystal data

$\mathrm{C}_{7} \mathrm{H}_{11} \mathrm{~N}_{2}{ }^{+} \cdot \mathrm{C}_{7} \mathrm{H}_{5} \mathrm{O}_{2}{ }^{-} \quad M_{r}=244.29$

† Additional correspondence author, e-mail: habibah@ipharm.gov.my or habibahw@usm.my. Institute of Pharmaceutical and Neutraceuticals, Malaysia Ministry of Science and Technology and Innovation Science Complex, 11900, Penang, Malaysia.

§ Thomson Reuters ResearcherID: A-3561-2009.
Monoclinic, $P 2_{1} / c$

$a=7.5362(16) \AA$

$b=22.937(4) \AA$

$c=8.2124(14) \AA$

$\beta=109.820(2)^{\circ}$

$V=1335.5$ (4) $\AA^{3}$

Data collection

Bruker SMART APEXII CCD area-detector diffractometer Absorption correction: multi-scan (SADABS;p Bruker, 2009) $T_{\min }=0.954, T_{\max }=0.996$

Refinement

$R\left[F^{2}>2 \sigma\left(F^{2}\right)\right]=0.044$

$w R\left(F^{2}\right)=0.127$

$S=1.02$

2336 reflections

199 parameters
$Z=4$

Mo $K \alpha$ radiation

$\mu=0.08 \mathrm{~mm}^{-1}$

$T=296 \mathrm{~K}$

$0.57 \times 0.23 \times 0.05 \mathrm{~mm}$

7639 measured reflections 2336 independent reflections 1527 reflections with $I>2 \sigma(I)$ $R_{\text {int }}=0.029$

$\mathrm{H}$ atoms treated by a mixture of independent and constrained refinement

$\Delta \rho_{\max }=0.10 \mathrm{e}^{-3}$

$\Delta \rho_{\min }=-0.14{\mathrm{e} \AA^{-3}}^{-3}$

Table 1

Hydrogen-bond geometry $\left(\AA{ }^{\circ}\right)$.

\begin{tabular}{lllll}
\hline$D-\mathrm{H} \cdots A$ & $D-\mathrm{H}$ & $\mathrm{H} \cdots A$ & $D \cdots A$ & $D-\mathrm{H} \cdots A$ \\
\hline $\mathrm{N} 1-\mathrm{H} 1 N 1 \cdots \mathrm{O} 2$ & 1.04 & 1.65 & $2.683(2)$ & 172 \\
$\mathrm{~N} 2-\mathrm{H} 1 N 2 \cdots \mathrm{O} 1$ & 1.01 & 1.78 & $2.779(2)$ & 171 \\
$\mathrm{~N} 2-\mathrm{H} 2 N 2 \cdots \mathrm{O} 1{ }^{\mathrm{i}}$ & 0.90 & 1.97 & $2.853(2)$ & 168 \\
\hline
\end{tabular}

Symmetry code: (i) $x,-y+\frac{1}{2}, z-\frac{1}{2}$.

Data collection: APEX2 (Bruker, 2009); cell refinement: SAINT (Bruker, 2009); data reduction: $S A I N T$; $\operatorname{program}(\mathrm{s})$ used to solve structure: SHELXTL (Sheldrick, 2008); program(s) used to refine structure: SHELXTL; molecular graphics: SHELXTL; software used to prepare material for publication: SHELXTL and PLATON (Spek, 2009).

This research was supported by Universiti Sains Malaysia (USM) under the University Research grant (No. 1001/ PFARMASI/815004) and the Ministry of Science, Technology and Innovation through an R\&D Initiative Grant (09-05-IFNMEB 004). HKF and MMR also thank USM for the Research University Grant (No. 1001/PFIZIK/811160). MRA gratefully acknowledges a PhD scholarship from Universiti Malaysia Sarawak.

Supplementary data and figures for this paper are available from the IUCr electronic archives (Reference: FJ2327).

\title{
References
}

Bernstein, J., Davis, R. E., Shimoni, L. \& Chang, N.-L. (1995). Angew. Chem. Int. Ed. Engl. 34, 1555-1573.

Bruker (2009). APEX2, SAINT and SADABS. Bruker AXS Inc., Madison, Wisconsin, USA.

Dhar, D. N. \& Taploo, C. L. (1982). J. Sci. Ind. Res. 41, 501-506.

Jeffrey, G. A. (1997). An Introduction to Hydrogen Bonding. Oxford University Press.

Jeffrey, G. A. \& Saenger, W. (1991). Hydrogen Bonding in Biological Structures. Berlin: Springer.

Sheldrick, G. M. (2008). Acta Cryst. A64, 112-122.

Spek, A. L. (2009). Acta Cryst. D65, 148-155. 


\section{supporting information}

Acta Cryst. (2010). E66, o2496 [doi:10.1107/S1600536810034811]

\section{2-Amino-4,6-dimethylpyridinium benzoate}

\section{Mohd Razip Asaruddin, Habibah A Wahab, Nornisah Mohamed, Mohd Mustaqim Rosli and Hoong-Kun Fun}

\section{S1. Comment}

This compound is derived from 2-amino-4,6-dimethylpyridine and benzaldehyde. Schiff bases provide more potential sites for both chemical and biological activities of compounds. Schiff bases with azomethine linkage were used as antiinfectious agents (Dhar et al., 1982). Pyridine and its derivatives play an important role in heterocyclic chemistry (Jeffrey, 1997). They are often involved in hydrogen-bond interactions (Jeffrey \& Saenger, 1991).

The title 1:1 adduct compound contains an 2-amino-4,6-dimethylpyridinium cation and benzoate anion in the asymmetric unit. The parameters in (I), (Fig. 1), are within normal ranges. The 2-amino-4,6-dimethylpyridinium cation is planar with the maximum deviation of 0.005 (2) $\AA$ for atom C9. The $\mathrm{H}$ atoms of the methyl groups are disordered over two positions and with fixed site-occupancy factors of 0.50:0.50 for both of the methyl groups. The carboxylate group in benzoate anion is slightly twisted and make a dihedral angle of $7.2(1)^{\circ}$ with the attached benzene ring.

The 2-amino-4,6-dimethylpyridinium cation and benzoate anion groups are linked together by intermolecular N1$\mathrm{H} 1 \mathrm{~N} 1 \cdots \mathrm{O} 2$ and $\mathrm{N} 2-\mathrm{H} 1 \mathrm{~N} 2 \cdots \mathrm{O} 1$ interactions (Table 1$)$ forming an $\mathrm{R}^{2}{ }_{2}(8)$ ring motif. In the crystal structure, the molecules stabilized by intermolecular $\mathrm{N}-\mathrm{H} \cdots \mathrm{O}$ hydrogen bonds (Table 1 ) and $\pi-\pi$ interactions with $\mathrm{Cg} 1-\mathrm{Cg} 2=$ $3.661(2) \AA(\mathrm{Cg} 1=\mathrm{N} 1 / \mathrm{C} 8-\mathrm{C} 12, \mathrm{Cg} 2=\mathrm{C} 1-\mathrm{C} 6)$.

\section{S2. Experimental}

An ethanol solution $(20 \mathrm{ml})$ of 2-amino-4,6-dimethylpyridine (1.22 g, Aldrich) and benzaldehyde (1.06 g, Merck) were mixed, heated on a hot plate and stirred with a magnetic stirrer. The reaction mixture was refluxed for $4 \mathrm{~h}$. The resulting condensation solution was allowed to cool slowly at room temperature to form brownish materials. Purification was done using thin layer chromatography (TLC) and silica gel column chromatography (CC) eluted by chloroform:methanol and n-hexane:ethyl acetate solvent system. Finally the pure compound was recrystallized in ethanol which afforded the $\mathrm{C}_{7} \mathrm{H}_{11} \mathrm{~N}^{+} . \mathrm{C}_{7} \mathrm{H}_{5} \mathrm{O}_{2}^{-}$salt.

\section{S3. Refinement}

$\mathrm{N}$ bound $\mathrm{H}$ atoms was located from a difference Fourier map and were refined using a riding model, with $\mathrm{U}_{\text {iso }}(\mathrm{H})=$ $1.2 \mathrm{U}_{\mathrm{eq}}(\mathrm{N})$. The methyl hydrogen atoms were located from the difference Fourier map and refined freely with the parent atom $\left[\mathrm{U}_{\mathrm{iso}}(\mathrm{H})=1.5 \mathrm{U}_{\mathrm{eq}}(\mathrm{C})\right]$. The rest of the hydrogen atoms were positioned geometrically and refined as riding model $\left[\mathrm{U}_{\mathrm{iso}}(\mathrm{H})=1.2 \mathrm{U}_{\mathrm{eq}}(\mathrm{C})\right]$. 


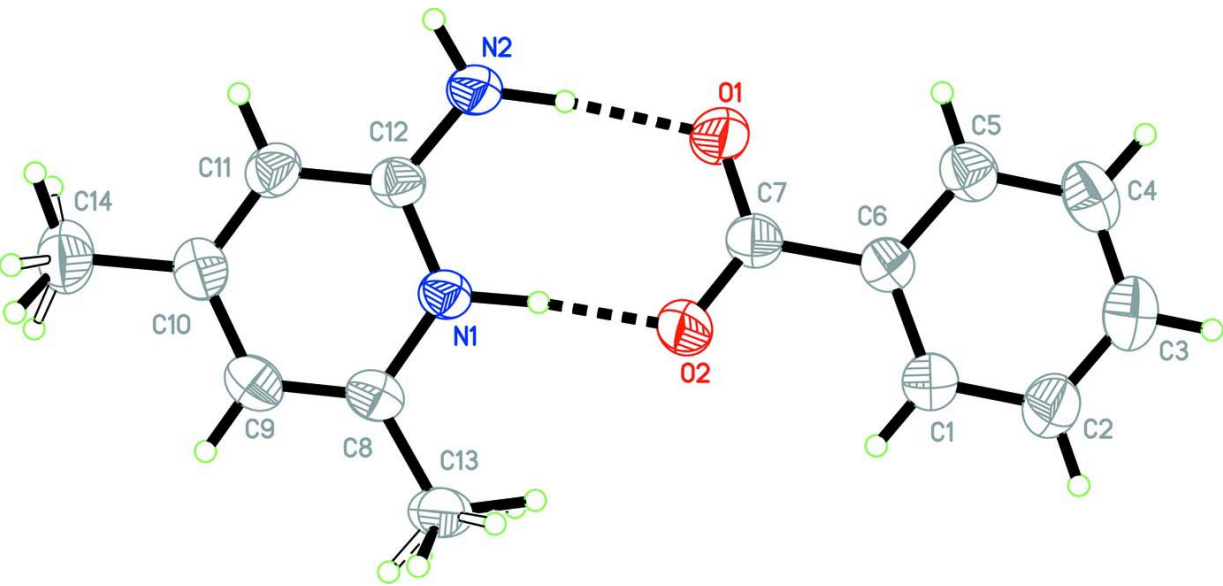

\section{Figure 1}

The molecular structure, showing 30\% probability displacement ellipsoids and the atom-numbering scheme. Hydrogen atoms are shown as spheres of arbitrary radius. Dashed lines indicate hydrogen bonds.

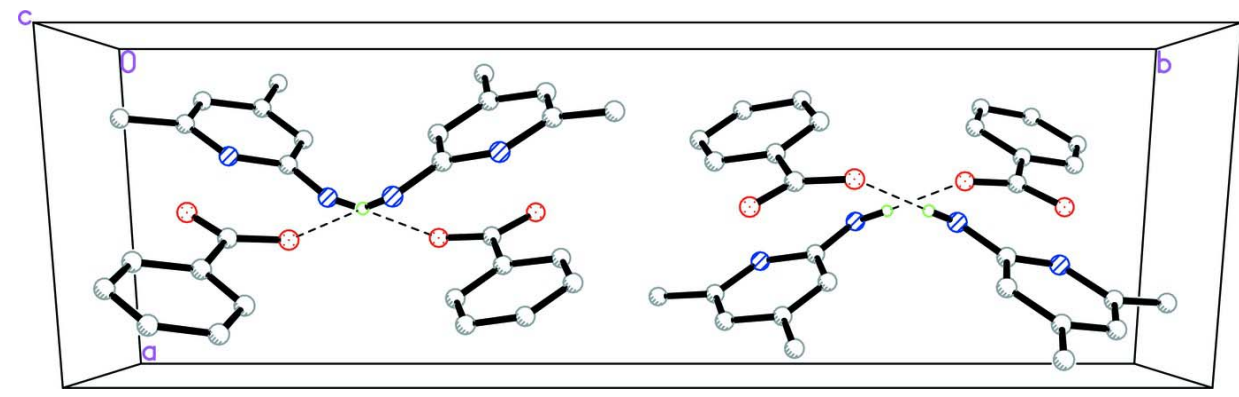

\section{Figure 2}

The crystal packing of (I) viewed along the $\mathrm{c}$ axis. Dashed lines indicate hydrogen bonds. $\mathrm{H}$ atoms not involved in the hydrogen bond interactions have been omitted for clarity.

\section{2-Amino-4,6-dimethylpyridinium benzoate}

Crystal data

$\mathrm{C}_{7} \mathrm{H}_{11} \mathrm{~N}_{2}^{+} \cdot \mathrm{C}_{7} \mathrm{H}_{5} \mathrm{O}_{2}^{-}$

$M_{r}=244.29$

Monoclinic, $P 2_{1} / c$

Hall symbol: $-\mathrm{P} 2 \mathrm{ybc}$

$a=7.5362(16) \AA$

$b=22.937(4) \AA$

$c=8.2124(14) \AA$

$\beta=109.820(2)^{\circ}$

$V=1335.5(4) \AA^{3}$

$Z=4$

\section{Data collection}

Bruker SMART APEXII CCD area-detector diffractometer

Radiation source: fine-focus sealed tube

Graphite monochromator

$\varphi$ and $\omega$ scans
$F(000)=520$

$D_{\mathrm{x}}=1.215 \mathrm{Mg} \mathrm{m}^{-3}$

Mo $K \alpha$ radiation, $\lambda=0.71073 \AA$

Cell parameters from 1881 reflections

$\theta=2.8-30.1^{\circ}$

$\mu=0.08 \mathrm{~mm}^{-1}$

$T=296 \mathrm{~K}$

Plate, colourless

$0.57 \times 0.23 \times 0.05 \mathrm{~mm}$

Absorption correction: multi-scan

(SADABS;p Bruker, 2009)

$T_{\min }=0.954, T_{\max }=0.996$

7639 measured reflections

2336 independent reflections

1527 reflections with $I>2 \sigma(I)$ 


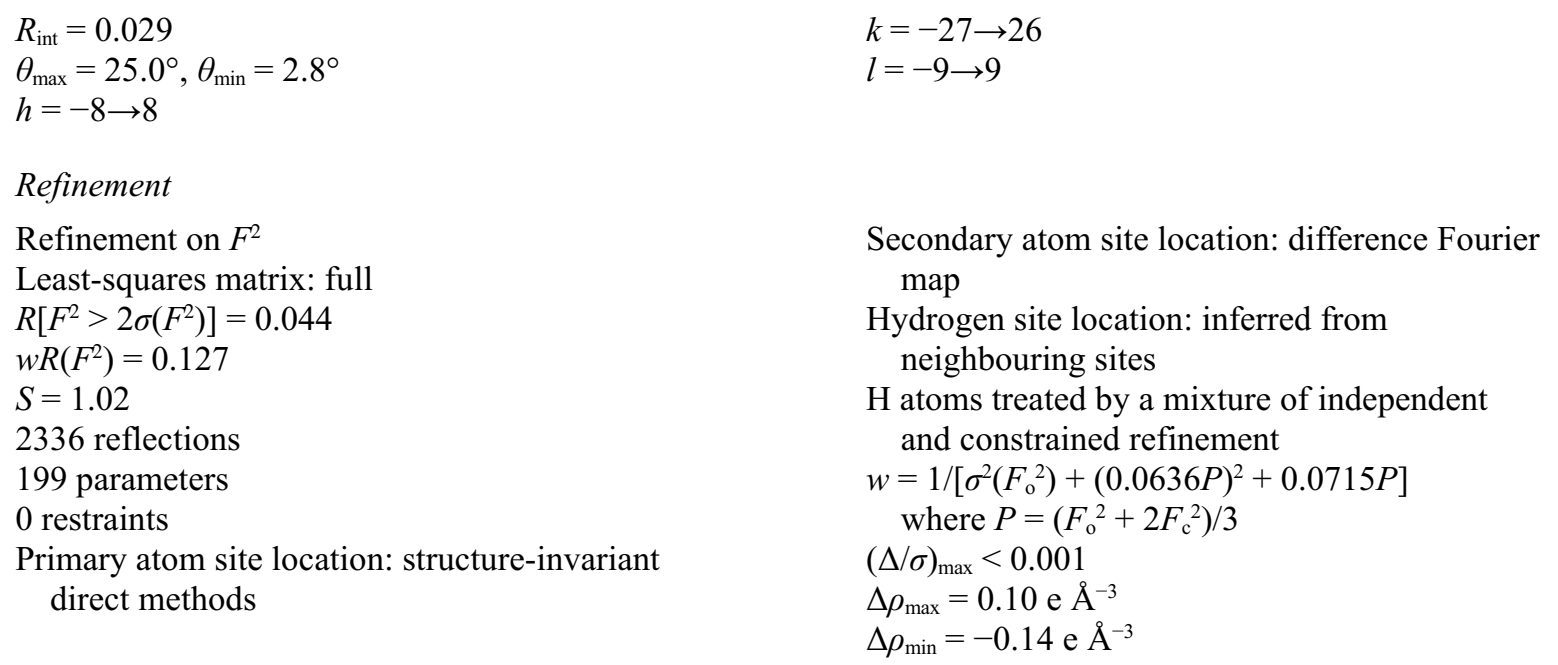

Special details

Geometry. All esds (except the esd in the dihedral angle between two l.s. planes) are estimated using the full covariance matrix. The cell esds are taken into account individually in the estimation of esds in distances, angles and torsion angles; correlations between esds in cell parameters are only used when they are defined by crystal symmetry. An approximate (isotropic) treatment of cell esds is used for estimating esds involving 1.s. planes.

Refinement. Refinement of $\mathrm{F}^{2}$ against ALL reflections. The weighted $\mathrm{R}$-factor $\mathrm{wR}$ and goodness of fit $\mathrm{S}$ are based on $\mathrm{F}^{2}$, conventional R-factors $\mathrm{R}$ are based on $\mathrm{F}$, with $\mathrm{F}$ set to zero for negative $\mathrm{F}^{2}$. The threshold expression of $\mathrm{F}^{2}>2$ sigma $\left(\mathrm{F}^{2}\right)$ is used only for calculating R-factors ( $\mathrm{gt}$ ) etc. and is not relevant to the choice of reflections for refinement. R-factors based on $\mathrm{F}^{2}$ are statistically about twice as large as those based on F, and R- factors based on ALL data will be even larger.

Fractional atomic coordinates and isotropic or equivalent isotropic displacement parameters $\left(\AA^{2}\right)$

\begin{tabular}{|c|c|c|c|c|c|}
\hline & $x$ & $y$ & $z$ & $U_{\text {iso }} * / U_{\text {eq }}$ & Occ. $(<1)$ \\
\hline O1 & $0.5868(2)$ & $0.30973(6)$ & $0.23891(18)$ & $0.0874(5)$ & \\
\hline $\mathrm{O} 2$ & $0.5079(2)$ & $0.40213(6)$ & $0.18422(17)$ & $0.0833(5)$ & \\
\hline $\mathrm{C} 1$ & $0.6431(3)$ & $0.42956(9)$ & 0.5357 (3) & $0.0703(5)$ & \\
\hline H1A & 0.5807 & 0.4584 & 0.4578 & $0.084 *$ & \\
\hline $\mathrm{C} 2$ & 0.7115 & $0.44196(11)$ & 0.7090 & $0.0868(6)$ & \\
\hline $\mathrm{H} 2 \mathrm{~A}$ & 0.6943 & 0.4789 & 0.7480 & $0.104 *$ & \\
\hline $\mathrm{C} 3$ & $0.8046(3)$ & $0.40029(12)$ & 0.8240 & $0.0924(7)$ & \\
\hline $\mathrm{H} 3 \mathrm{~A}$ & 0.8500 & 0.4087 & 0.9418 & $0.111^{*}$ & \\
\hline $\mathrm{C} 4$ & $0.8316(3)$ & $0.34597(11)$ & $0.7670(3)$ & $0.0872(7)$ & \\
\hline $\mathrm{H} 4 \mathrm{~A}$ & 0.8968 & 0.3177 & 0.8460 & $0.105^{*}$ & \\
\hline C5 & $0.7621(3)$ & $0.33309(9)$ & $0.5921(3)$ & $0.0722(5)$ & \\
\hline H5A & 0.7807 & 0.2962 & 0.5535 & $0.087^{*}$ & \\
\hline C6 & $0.6651(2)$ & $0.37496(8)$ & $0.4747(2)$ & $0.0583(5)$ & \\
\hline $\mathrm{C} 7$ & $0.5808(3)$ & $0.36135(8)$ & $0.2857(2)$ & $0.0635(5)$ & \\
\hline N1 & 0.33910 (19) & $0.38091(6)$ & $-0.15464(18)$ & $0.0574(4)$ & \\
\hline H1N1 & 0.3994 & 0.3858 & -0.0211 & $0.069 *$ & \\
\hline N2 & $0.4633(2)$ & $0.28852(6)$ & $-0.1151(2)$ & $0.0734(5)$ & \\
\hline $\mathrm{H} 1 \mathrm{~N} 2$ & 0.5218 & 0.2955 & 0.0136 & $0.088^{*}$ & \\
\hline $\mathrm{H} 2 \mathrm{~N} 2$ & 0.4965 & 0.2603 & -0.1752 & $0.088^{*}$ & \\
\hline $\mathrm{C} 8$ & $0.2395(2)$ & $0.42572(7)$ & $-0.2537(2)$ & $0.0616(5)$ & \\
\hline $\mathrm{C} 9$ & $0.1624(3)$ & $0.41807(9)$ & $-0.4262(3)$ & $0.0703(5)$ & \\
\hline
\end{tabular}




\begin{tabular}{|c|c|c|c|c|c|}
\hline H9A & 0.0956 & 0.4485 & -0.4947 & $0.084 *$ & \\
\hline $\mathrm{C} 10$ & 0.1811 (3) & $0.36533(9)$ & $-0.5041(2)$ & $0.0692(5)$ & \\
\hline $\mathrm{C} 11$ & $0.2816(3)$ & $0.32162(8)$ & $-0.4016(2)$ & $0.0660(5)$ & \\
\hline H11A & 0.2959 & 0.2863 & -0.4511 & $0.079 *$ & \\
\hline $\mathrm{C} 12$ & $0.3632(3)$ & $0.32926(7)$ & $-0.2236(2)$ & $0.0582(5)$ & \\
\hline $\mathrm{C} 13$ & $0.2269(4)$ & $0.47999(10)$ & $-0.1583(4)$ & $0.0817(7)$ & \\
\hline H13A & $0.086(9)$ & $0.490(3)$ & $-0.181(9)$ & $0.123 *$ & 0.50 \\
\hline H13B & $0.285(10)$ & 0.475 & $-0.023(9)$ & $0.123^{*}$ & 0.50 \\
\hline $\mathrm{H} 13 \mathrm{C}$ & $0.298(10)$ & $0.513(2)$ & $-0.195(8)$ & $0.123^{*}$ & 0.50 \\
\hline H13D & 0.155 (12) & $0.506(3)$ & $-0.232(7)$ & $0.123^{*}$ & 0.50 \\
\hline H13E & $0.344(8)$ & $0.494(3)$ & $-0.087(10)$ & $0.123^{*}$ & 0.50 \\
\hline H13F & $0.184(10)$ & $0.470(3)$ & $-0.045(10)$ & $0.123 *$ & 0.50 \\
\hline C14 & $0.0940(5)$ & 0.3577 (2) & $-0.6963(3)$ & $0.0984(8)$ & \\
\hline H14A & $0.143(11)$ & $0.323(5)$ & $-0.738(10)$ & $0.148^{*}$ & 0.50 \\
\hline H14B & 0.117 (14) & $0.392(3)$ & $-0.758(11)$ & $0.148^{*}$ & 0.50 \\
\hline $\mathrm{H} 14 \mathrm{C}$ & $-0.041(12)$ & $0.347(3)$ & $-0.724(9)$ & $0.148^{*}$ & 0.50 \\
\hline H14D & $0.071(13)$ & $0.319(5)$ & $-0.722(11)$ & $0.148^{*}$ & 0.50 \\
\hline H14E & $0.179(10)$ & $0.376(5)$ & $-0.759(10)$ & $0.148^{*}$ & 0.50 \\
\hline $\mathrm{H} 14 \mathrm{~F}$ & $-0.037(11)$ & $0.382(3)$ & $-0.749(9)$ & $0.148^{*}$ & 0.50 \\
\hline
\end{tabular}

Atomic displacement parameters $\left(\AA^{2}\right)$

\begin{tabular}{lllllll}
\hline & $U^{11}$ & $U^{22}$ & $U^{33}$ & $U^{12}$ & $U^{13}$ & $U^{23}$ \\
\hline $\mathrm{O} 1$ & $0.1331(13)$ & $0.0527(8)$ & $0.0720(9)$ & $-0.0037(8)$ & $0.0288(8)$ & $0.0009(7)$ \\
$\mathrm{O} 2$ & $0.1135(12)$ & $0.0558(8)$ & $0.0663(9)$ & $-0.0009(7)$ & $0.0119(8)$ & $0.0059(7)$ \\
$\mathrm{C} 1$ & $0.0710(12)$ & $0.0710(13)$ & $0.0675(13)$ & $0.0012(10)$ & $0.0216(10)$ & $-0.0006(10)$ \\
$\mathrm{C} 2$ & $0.0918(16)$ & $0.0927(16)$ & $0.0724(15)$ & $0.0031(12)$ & $0.0231(12)$ & $-0.0119(13)$ \\
$\mathrm{C} 3$ & $0.0939(16)$ & $0.114(2)$ & $0.0648(14)$ & $-0.0117(14)$ & $0.0210(12)$ & $-0.0052(14)$ \\
$\mathrm{C} 4$ & $0.0785(14)$ & $0.0990(18)$ & $0.0745(15)$ & $-0.0042(12)$ & $0.0135(11)$ & $0.0235(13)$ \\
$\mathrm{C} 5$ & $0.0718(12)$ & $0.0666(12)$ & $0.0748(14)$ & $-0.0054(9)$ & $0.0204(10)$ & $0.0104(10)$ \\
$\mathrm{C} 6$ & $0.0551(10)$ & $0.0569(11)$ & $0.0638(11)$ & $-0.0076(8)$ & $0.0212(8)$ & $0.0039(9)$ \\
$\mathrm{C} 7$ & $0.0713(12)$ & $0.0520(11)$ & $0.0673(12)$ & $-0.0098(9)$ & $0.0238(9)$ & $0.0053(10)$ \\
$\mathrm{N} 1$ & $0.0639(9)$ & $0.0481(8)$ & $0.0611(9)$ & $-0.0003(7)$ & $0.0223(7)$ & $0.0034(7)$ \\
$\mathrm{N} 2$ & $0.1069(13)$ & $0.0532(9)$ & $0.0645(10)$ & $0.0139(8)$ & $0.0348(9)$ & $0.0070(8)$ \\
$\mathrm{C} 8$ & $0.0552(10)$ & $0.0549(11)$ & $0.0730(13)$ & $0.0005(8)$ & $0.0192(9)$ & $0.0079(9)$ \\
$\mathrm{C} 9$ & $0.0616(11)$ & $0.0704(13)$ & $0.0755(14)$ & $0.0094(9)$ & $0.0187(10)$ & $0.0136(10)$ \\
$\mathrm{C} 10$ & $0.0580(11)$ & $0.0871(14)$ & $0.0641(12)$ & $0.0035(10)$ & $0.0228(9)$ & $0.0068(11)$ \\
$\mathrm{C} 11$ & $0.0721(12)$ & $0.0667(12)$ & $0.0664(12)$ & $0.0017(9)$ & $0.0328(10)$ & $-0.0042(9)$ \\
$\mathrm{C} 12$ & $0.0643(11)$ & $0.0513(10)$ & $0.0650(12)$ & $0.0000(8)$ & $0.0297(9)$ & $0.0049(9)$ \\
$\mathrm{C} 13$ & $0.0840(17)$ & $0.0544(13)$ & $0.0937(19)$ & $0.0083(11)$ & $0.0132(14)$ & $-0.0013(12)$ \\
$\mathrm{C} 14$ & $0.093(2)$ & $0.130(3)$ & $0.0671(15)$ & $0.014(2)$ & $0.0203(14)$ & $0.0021(16)$ \\
& & & & & &
\end{tabular}

Geometric parameters $\left(\AA,{ }^{\circ}\right)$

\begin{tabular}{llll}
\hline $\mathrm{O} 1-\mathrm{C} 7$ & $1.250(2)$ & $\mathrm{C} 8-\mathrm{C} 9$ & $1.348(3)$ \\
$\mathrm{O} 2-\mathrm{C} 7$ & $1.249(2)$ & $\mathrm{C} 8-\mathrm{C} 13$ & $1.491(3)$ \\
$\mathrm{C} 1-\mathrm{C} 2$ & $1.369(3)$ & $\mathrm{C} 9-\mathrm{C} 10$ & $1.398(3)$ \\
$\mathrm{C} 1-\mathrm{C} 6$ & $1.380(3)$ & $\mathrm{C} 9-\mathrm{H} 9 \mathrm{~A}$ & 0.9300
\end{tabular}




\begin{tabular}{|c|c|c|c|}
\hline $\mathrm{C} 1-\mathrm{H} 1 \mathrm{~A}$ & 0.9300 & $\mathrm{C} 10-\mathrm{C} 11$ & $1.362(3)$ \\
\hline $\mathrm{C} 2-\mathrm{C} 3$ & $1.360(3)$ & $\mathrm{C} 10-\mathrm{C} 14$ & $1.500(3)$ \\
\hline $\mathrm{C} 2-\mathrm{H} 2 \mathrm{~A}$ & 0.9300 & $\mathrm{C} 11-\mathrm{C} 12$ & $1.391(3)$ \\
\hline $\mathrm{C} 3-\mathrm{C} 4$ & $1.370(3)$ & $\mathrm{C} 11-\mathrm{H} 11 \mathrm{~A}$ & 0.9300 \\
\hline $\mathrm{C} 3-\mathrm{H} 3 \mathrm{~A}$ & 0.9300 & $\mathrm{C} 13-\mathrm{H} 13 \mathrm{~A}$ & $1.04(6)$ \\
\hline $\mathrm{C} 4-\mathrm{C} 5$ & $1.384(3)$ & $\mathrm{C} 13-\mathrm{H} 13 \mathrm{~B}$ & $1.06(7)$ \\
\hline $\mathrm{C} 4-\mathrm{H} 4 \mathrm{~A}$ & 0.9300 & $\mathrm{C} 13-\mathrm{H} 13 \mathrm{C}$ & $1.04(5)$ \\
\hline $\mathrm{C} 5-\mathrm{C} 6$ & $1.381(3)$ & $\mathrm{C} 13-\mathrm{H} 13 \mathrm{D}$ & $0.89(6)$ \\
\hline $\mathrm{C} 5-\mathrm{H} 5 \mathrm{~A}$ & 0.9300 & $\mathrm{C} 13-\mathrm{H} 13 \mathrm{E}$ & $0.93(6)$ \\
\hline $\mathrm{C} 6-\mathrm{C} 7$ & $1.497(3)$ & $\mathrm{C} 13-\mathrm{H} 13 \mathrm{~F}$ & $1.11(7)$ \\
\hline $\mathrm{N} 1-\mathrm{C} 12$ & $1.352(2)$ & $\mathrm{C} 14-\mathrm{H} 14 \mathrm{~A}$ & $0.99(9)$ \\
\hline $\mathrm{N} 1-\mathrm{C} 8$ & $1.367(2)$ & C14-H14B & $0.98(10)$ \\
\hline $\mathrm{N} 1-\mathrm{H} 1 \mathrm{~N} 1$ & 1.0414 & $\mathrm{C} 14-\mathrm{H} 14 \mathrm{C}$ & $1.00(8)$ \\
\hline $\mathrm{N} 2-\mathrm{C} 12$ & $1.335(2)$ & C14-H14D & $0.90(10)$ \\
\hline $\mathrm{N} 2-\mathrm{H} 1 \mathrm{~N} 2$ & 1.0101 & C14-H14E & $1.03(10)$ \\
\hline $\mathrm{N} 2-\mathrm{H} 2 \mathrm{~N} 2$ & 0.9002 & $\mathrm{C} 14-\mathrm{H} 14 \mathrm{~F}$ & $1.09(7)$ \\
\hline $\mathrm{C} 2-\mathrm{C} 1-\mathrm{C} 6$ & $121.1(2)$ & $\mathrm{N} 1-\mathrm{C} 12-\mathrm{C} 11$ & $118.47(16)$ \\
\hline $\mathrm{C} 2-\mathrm{C} 1-\mathrm{H} 1 \mathrm{~A}$ & 119.4 & $\mathrm{C} 8-\mathrm{C} 13-\mathrm{H} 13 \mathrm{~A}$ & $109(3)$ \\
\hline $\mathrm{C} 6-\mathrm{C} 1-\mathrm{H} 1 \mathrm{~A}$ & 119.4 & $\mathrm{C} 8-\mathrm{C} 13-\mathrm{H} 13 \mathrm{~B}$ & $112(3)$ \\
\hline $\mathrm{C} 3-\mathrm{C} 2-\mathrm{C} 1$ & $120.0(2)$ & $\mathrm{H} 13 \mathrm{~A}-\mathrm{C} 13-\mathrm{H} 13 \mathrm{~B}$ & $104(4)$ \\
\hline $\mathrm{C} 3-\mathrm{C} 2-\mathrm{H} 2 \mathrm{~A}$ & 120.0 & $\mathrm{C} 8-\mathrm{C} 13-\mathrm{H} 13 \mathrm{C}$ & $109(3)$ \\
\hline $\mathrm{C} 1-\mathrm{C} 2-\mathrm{H} 2 \mathrm{~A}$ & 120.0 & $\mathrm{H} 13 \mathrm{~A}-\mathrm{C} 13-\mathrm{H} 13 \mathrm{C}$ & $113(4)$ \\
\hline $\mathrm{C} 2-\mathrm{C} 3-\mathrm{C} 4$ & $120.2(2)$ & $\mathrm{H} 13 \mathrm{~B}-\mathrm{C} 13-\mathrm{H} 13 \mathrm{C}$ & $109(4)$ \\
\hline $\mathrm{C} 2-\mathrm{C} 3-\mathrm{H} 3 \mathrm{~A}$ & 119.9 & $\mathrm{C} 8-\mathrm{C} 13-\mathrm{H} 13 \mathrm{D}$ & $109(4)$ \\
\hline $\mathrm{C} 4-\mathrm{C} 3-\mathrm{H} 3 \mathrm{~A}$ & 119.9 & $\mathrm{H} 13 \mathrm{~A}-\mathrm{C} 13-\mathrm{H} 13 \mathrm{D}$ & $52(4)$ \\
\hline $\mathrm{C} 3-\mathrm{C} 4-\mathrm{C} 5$ & $120.1(2)$ & $\mathrm{H} 13 \mathrm{~B}-\mathrm{C} 13-\mathrm{H} 13 \mathrm{D}$ & $137(5)$ \\
\hline $\mathrm{C} 3-\mathrm{C} 4-\mathrm{H} 4 \mathrm{~A}$ & 120.0 & $\mathrm{H} 13 \mathrm{C}-\mathrm{C} 13-\mathrm{H} 13 \mathrm{D}$ & $65(4)$ \\
\hline $\mathrm{C} 5-\mathrm{C} 4-\mathrm{H} 4 \mathrm{~A}$ & 120.0 & $\mathrm{C} 8-\mathrm{C} 13-\mathrm{H} 13 \mathrm{E}$ & $113(4)$ \\
\hline $\mathrm{C} 6-\mathrm{C} 5-\mathrm{C} 4$ & $120.0(2)$ & $\mathrm{H} 13 \mathrm{~A}-\mathrm{C} 13-\mathrm{H} 13 \mathrm{E}$ & $137(4)$ \\
\hline $\mathrm{C} 6-\mathrm{C} 5-\mathrm{H} 5 \mathrm{~A}$ & 120.0 & $\mathrm{H} 13 \mathrm{~B}-\mathrm{C} 13-\mathrm{H} 13 \mathrm{E}$ & $54(4)$ \\
\hline $\mathrm{C} 4-\mathrm{C} 5-\mathrm{H} 5 \mathrm{~A}$ & 120.0 & $\mathrm{H} 13 \mathrm{C}-\mathrm{C} 13-\mathrm{H} 13 \mathrm{E}$ & $57(4)$ \\
\hline $\mathrm{C} 1-\mathrm{C} 6-\mathrm{C} 5$ & $118.58(18)$ & $\mathrm{H} 13 \mathrm{D}-\mathrm{C} 13-\mathrm{H} 13 \mathrm{E}$ & $116(5)$ \\
\hline $\mathrm{C} 1-\mathrm{C} 6-\mathrm{C} 7$ & $120.30(16)$ & $\mathrm{C} 8-\mathrm{C} 13-\mathrm{H} 13 \mathrm{~F}$ & $111(3)$ \\
\hline $\mathrm{C} 5-\mathrm{C} 6-\mathrm{C} 7$ & $121.10(17)$ & $\mathrm{H} 13 \mathrm{~A}-\mathrm{C} 13-\mathrm{H} 13 \mathrm{~F}$ & $68(4)$ \\
\hline $\mathrm{O} 2-\mathrm{C} 7-\mathrm{O} 1$ & $123.88(17)$ & $\mathrm{H} 13 \mathrm{C}-\mathrm{C} 13-\mathrm{H} 13 \mathrm{~F}$ & $136(4)$ \\
\hline $\mathrm{O} 2-\mathrm{C} 7-\mathrm{C} 6$ & $118.14(16)$ & $\mathrm{H} 13 \mathrm{D}-\mathrm{C} 13-\mathrm{H} 13 \mathrm{~F}$ & $115(5)$ \\
\hline $\mathrm{O} 1-\mathrm{C} 7-\mathrm{C} 6$ & $117.98(16)$ & $\mathrm{H} 13 \mathrm{E}-\mathrm{C} 13-\mathrm{H} 13 \mathrm{~F}$ & $92(5)$ \\
\hline $\mathrm{C} 12-\mathrm{N} 1-\mathrm{C} 8$ & $122.30(16)$ & $\mathrm{C} 10-\mathrm{C} 14-\mathrm{H} 14 \mathrm{~A}$ & $112(5)$ \\
\hline $\mathrm{C} 12-\mathrm{N} 1-\mathrm{H} 1 \mathrm{~N} 1$ & 117.7 & $\mathrm{C} 10-\mathrm{C} 14-\mathrm{H} 14 \mathrm{~B}$ & $111(5)$ \\
\hline $\mathrm{C} 8-\mathrm{N} 1-\mathrm{H} 1 \mathrm{~N} 1$ & 120.0 & $\mathrm{H} 14 \mathrm{~A}-\mathrm{C} 14-\mathrm{H} 14 \mathrm{~B}$ & $108(6)$ \\
\hline $\mathrm{C} 12-\mathrm{N} 2-\mathrm{H} 1 \mathrm{~N} 2$ & 122.4 & $\mathrm{C} 10-\mathrm{C} 14-\mathrm{H} 14 \mathrm{C}$ & $108(4)$ \\
\hline $\mathrm{C} 12-\mathrm{N} 2-\mathrm{H} 2 \mathrm{~N} 2$ & 109.7 & $\mathrm{H} 14 \mathrm{~A}-\mathrm{C} 14-\mathrm{H} 14 \mathrm{C}$ & $102(7)$ \\
\hline $\mathrm{H} 1 \mathrm{~N} 2-\mathrm{N} 2-\mathrm{H} 2 \mathrm{~N} 2$ & 125.7 & $\mathrm{H} 14 \mathrm{~B}-\mathrm{C} 14-\mathrm{H} 14 \mathrm{C}$ & $115(6)$ \\
\hline $\mathrm{C} 9-\mathrm{C} 8-\mathrm{N} 1$ & $118.79(17)$ & $\mathrm{C} 10-\mathrm{C} 14-\mathrm{H} 14 \mathrm{D}$ & $110(6)$ \\
\hline $\mathrm{C} 9-\mathrm{C} 8-\mathrm{C} 13$ & $125.41(18)$ & $\mathrm{H} 14 \mathrm{~B}-\mathrm{C} 14-\mathrm{H} 14 \mathrm{D}$ & $135(7)$ \\
\hline $\mathrm{N} 1-\mathrm{C} 8-\mathrm{C} 13$ & $115.79(18)$ & $\mathrm{H} 14 \mathrm{C}-\mathrm{C} 14-\mathrm{H} 14 \mathrm{D}$ & $67(5)$ \\
\hline $\mathrm{C} 8-\mathrm{C} 9-\mathrm{C} 10$ & $121.30(17)$ & $\mathrm{C} 10-\mathrm{C} 14-\mathrm{H} 14 \mathrm{E}$ & $111(4)$ \\
\hline
\end{tabular}




$\begin{array}{llll}\mathrm{C} 8-\mathrm{C} 9-\mathrm{H} 9 \mathrm{~A} & 119.4 & \mathrm{H} 14 \mathrm{~A}-\mathrm{C} 14-\mathrm{H} 14 \mathrm{E} & 77(5) \\ \mathrm{C} 10-\mathrm{C} 9-\mathrm{H} 9 \mathrm{~A} & 119.4 & \mathrm{H} 14 \mathrm{C}-\mathrm{C} 14-\mathrm{H} 14 \mathrm{E} & 138(5) \\ \mathrm{C} 11-\mathrm{C} 10-\mathrm{C} 9 & 118.37(18) & \mathrm{H} 14 \mathrm{D}-\mathrm{C} 14-\mathrm{H} 14 \mathrm{E} & 112(7) \\ \mathrm{C} 11-\mathrm{C} 10-\mathrm{C} 14 & 121.2(2) & \mathrm{C} 10-\mathrm{C} 14-\mathrm{H} 14 \mathrm{~F} & 112(3) \\ \mathrm{C} 9-\mathrm{C} 10-\mathrm{C} 14 & 120.5(2) & \mathrm{H} 14 \mathrm{~A}-\mathrm{C} 14-\mathrm{H} 14 \mathrm{~F} & 132(6) \\ \mathrm{C} 10-\mathrm{C} 11-\mathrm{C} 12 & 120.77(18) & \mathrm{H} 14 \mathrm{~B}-\mathrm{C} 14-\mathrm{H} 14 \mathrm{~F} & 72(5) \\ \mathrm{C} 10-\mathrm{C} 11-\mathrm{H} 11 \mathrm{~A} & 119.6 & \mathrm{H} 14 \mathrm{C}-\mathrm{C} 14-\mathrm{H} 14 \mathrm{~F} & 46(4) \\ \mathrm{C} 12-\mathrm{C} 11-\mathrm{H} 11 \mathrm{~A} & 119.6 & \mathrm{H} 14 \mathrm{D}-\mathrm{C} 14-\mathrm{H} 14 \mathrm{~F} & 109(7) \\ \mathrm{N} 2-\mathrm{C} 12-\mathrm{N} 1 & 117.34(16) & \mathrm{H} 14 \mathrm{E}-\mathrm{C} 14-\mathrm{H} 14 \mathrm{~F} & 104(6) \\ \mathrm{N} 2-\mathrm{C} 12-\mathrm{C} 11 & 124.19(17) & & \\ & & & \\ \mathrm{C} 6-\mathrm{C} 1-\mathrm{C} 2-\mathrm{C} 3 & 0.7(3) & \mathrm{C} 12-\mathrm{N} 1-\mathrm{C} 8-\mathrm{C} 9 & 0.1(2) \\ \mathrm{C} 1-\mathrm{C} 2-\mathrm{C} 3-\mathrm{C} 4 & 0.5(4) & \mathrm{C} 12-\mathrm{N} 1-\mathrm{C} 8-\mathrm{C} 13 & -179.6(2) \\ \mathrm{C} 2-\mathrm{C} 3-\mathrm{C} 4-\mathrm{C} 5 & -0.8(4) & \mathrm{N} 1-\mathrm{C} 8-\mathrm{C} 9-\mathrm{C} 10 & 0.7(3) \\ \mathrm{C} 3-\mathrm{C} 4-\mathrm{C} 5-\mathrm{C} 6 & -0.1(3) & \mathrm{C} 13-\mathrm{C} 8-\mathrm{C} 9-\mathrm{C} 10 & -179.7(2) \\ \mathrm{C} 2-\mathrm{C} 1-\mathrm{C} 6-\mathrm{C} 5 & -1.6(3) & \mathrm{C} 8-\mathrm{C} 9-\mathrm{C} 10-\mathrm{C} 11 & -0.8(3) \\ \mathrm{C} 2-\mathrm{C} 1-\mathrm{C} 6-\mathrm{C} 7 & 176.91(18) & \mathrm{C} 8-\mathrm{C} 9-\mathrm{C} 10-\mathrm{C} 14 & 179.9(2) \\ \mathrm{C} 4-\mathrm{C} 5-\mathrm{C} 6-\mathrm{C} 1 & 1.3(3) & \mathrm{C} 9-\mathrm{C} 10-\mathrm{C} 11-\mathrm{C} 12 & 0.2(3) \\ \mathrm{C} 4-\mathrm{C} 5-\mathrm{C} 6-\mathrm{C} 7 & -177.18(18) & \mathrm{C} 14-\mathrm{C} 10-\mathrm{C} 11-\mathrm{C} 12 & 179.5(2) \\ \mathrm{C} 1-\mathrm{C} 6-\mathrm{C} 7-\mathrm{O} 2 & 7.2(3) & \mathrm{C} 8-\mathrm{N} 1-\mathrm{C} 12-\mathrm{N} 2 & 179.88(16) \\ \mathrm{C} 5-\mathrm{C} 6-\mathrm{C} 7-\mathrm{O} 2 & -174.36(18) & \mathrm{C} 8-\mathrm{N} 1-\mathrm{C} 12-\mathrm{C} 11 & -0.6(2) \\ \mathrm{C} 1-\mathrm{C} 6-\mathrm{C} 7-\mathrm{O} 1 & -172.47(18) & \mathrm{C} 10-\mathrm{C} 11-\mathrm{C} 12-\mathrm{N} 2 & 179.94(18) \\ \mathrm{C} 5-\mathrm{C} 6-\mathrm{C} 7-\mathrm{O} 1 & 6.0(3) & \mathrm{C} 10-\mathrm{C} 11-\mathrm{C} 12-\mathrm{N} 1 & 0.5(3) \\ & & & \end{array}$

Hydrogen-bond geometry $\left(\AA,{ }^{\circ}\right)$

\begin{tabular}{lllll}
\hline$D-\mathrm{H}^{\cdots} A$ & $D-\mathrm{H}$ & $\mathrm{H} \cdots A$ & $D \cdots A$ & $D-\mathrm{H} \cdots A$ \\
$\mathrm{~N} 1-\mathrm{H} 1 N 1 \cdots \mathrm{O} 2$ & 1.04 & 1.65 & $2.683(2)$ & 172 \\
$\mathrm{~N} 2-\mathrm{H} 1 N 2 \cdots \mathrm{O} 1$ & 1.01 & 1.78 & $2.779(2)$ & 171 \\
$\mathrm{~N} 2-\mathrm{H} 2 N 2 \cdots \mathrm{O} 1^{\mathrm{i}}$ & 0.90 & 1.97 & $2.853(2)$ & 168 \\
\hline
\end{tabular}

Symmetry code: (i) $x,-y+1 / 2, z-1 / 2$. 